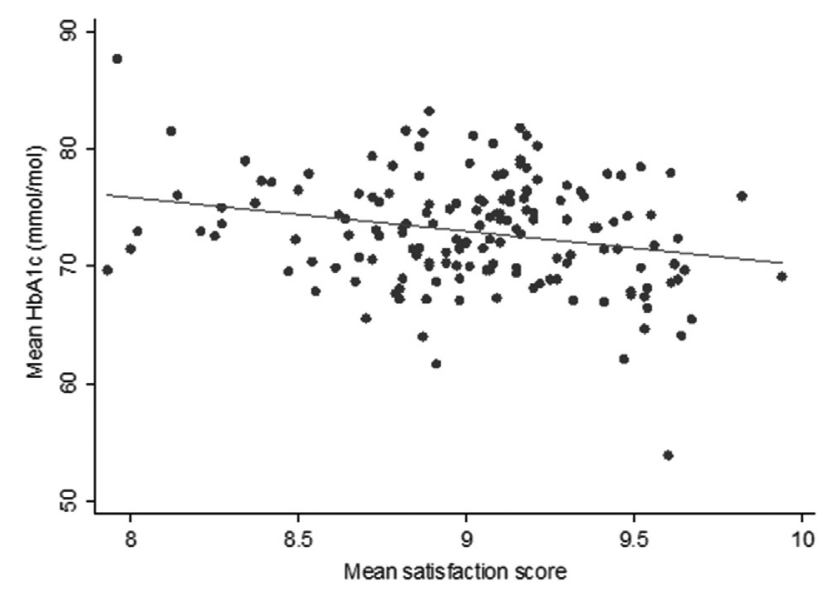

Abstract G474(P) Figure 2 Scatterplot with regression line for the association between mean satisfaction and mean $\mathrm{HbA} 1 \mathrm{c}$ at PDU level (2012-2013 NPDA data for England and Wales)

\section{G475 SUPPORTING PARENTS TO MANAGE CHRONIC CHILDHOOD CONDITIONS AT HOME: RESULTS OF A FEASIBILITY RANDOMISED CONTROLLED TRIAL OF A NEW INTERACTIVE HEALTH COMMUNICATION APPLICATION}

${ }^{1}$ VM Swallow, ${ }^{2} \mathrm{NJA}$ Webb, ${ }^{2} \mathrm{~T}$ Smith. ${ }^{1}$ School of Nursng, Midwifery and Social Work, University of Manchester, Manchester, UK; ${ }^{2}$ Paediatric Nephrology, Royal Manchester Children's Hospital, Manchester, UK

\subsection{6/archdischild-2015-308599.429}

Background Families living with chronic childhood conditions face multiple challenges and parents have previously identified the need for an interactive health communication application (IHCA) to improve their condition management ability. We developed, and evaluated in a feasibility randomised controlled trial the OPIS (online parent information and support) IHCA.

Methods Parents of children with chronic kidney diseases were randomly assigned to usual health-professional support for caregiving (control) or usual support plus password-protected access to OPIS for 20 weeks (intervention). We assessed feasibility descriptively in terms of recruitment and retention rates overall; assessed recruitment, retention, and uptake of OPIS and compared family condition management between groups using the Family Management Measure [FaMM] and qualitative interviews. Questionnaire data were analysed using descriptive statistics and qualitative data using Framework Analysis

Results 55 parents of 39 children were recruited. Three-quarters of intervention group parents $(19 / 26,73 \%)$ and control group parents $(22 / 29,76 \%)$ were retained, the overall retention rate was $41 / 55$ (75\%). The 41 parents completing the trial were asked to respond to the same 10 questionnaire scales at baseline and 20 weeks later; 10 scores were missing at baseline and nine were missing at 20 weeks. All intervention group parents accessed OPIS and showed a greater improvement in perceived competence to manage their child's condition compared to control group parents (adjusted mean FaMM Condition Management Ability Scale, intervention group 44.5 vs control group 41.9, difference $2.6,95 \%$ CI -1.6 to 6.7). Differences between groups agreed with qualitative findings that OPIS improved parents' management ability.

Conclusions OPIS is being made available as standard practice in the hospital where it was developed and evaluated. A full- scale national trial of the effectiveness of OPIS is feasible. Our design and methodology can be transferred to the management of other conditions in different contexts.

\section{G476 DIABETES MORTALITY TRENDS 1990 TO 2010 IN THE UK COMPARED WITH THE EU15 AND THE USA}

${ }^{1}$ RM Viner, ${ }^{1} \mathrm{R}$ Amin, ${ }^{1} \mathrm{D}$ Hargreaves, ${ }^{1} \mathrm{~A}$ Khanolkar, ${ }^{1} \mathrm{D}$ Charalampopoulos, ${ }^{2} \mathrm{~T}$ Candler ${ }^{3}$ I Wolfe, ${ }^{1} \mathrm{~T}$ Stephenson. ${ }^{1}$ Institute of Child Health, University College London, London, UK; ${ }^{2}$ Bristol Children's Hospital, Bristol, UK; ${ }^{3}$ Kings, Guys \& St. Thomas' Medical School, Kings College London, London, UK

\subsection{6/archdischild-2015-308599.430}

Aims We investigated whether the UK has higher child and youth diabetes mortality than in comparable European countries and the USA.

Methods We obtained data from the WHO World Mortality Database for the UK, the USA and the EU15+ (the 15 countries of the EU in 2004 plus Australia, Canada and Norway) for 1990 to 2010. Diabetes mortality rates were calculated for 1-14 and 15-24 year olds. Multilevel longitudinal Poisson regression models were constructed including all country-level data from 1990-2010 (378 country years) comparing the UK with the EU15+ and the USA. Graphics show average mortality across the EU15+.

Results Mortality trends are shown in Figure 1 for 1-14 year olds (both sexes) and Figure 2 (males) and Figure 3 (females) for 15-24 year olds. In 1990 the UK had higher mortality than the EU15 + amongst $1-14$ yo ( $p=0.004$ ) but not amongst $15-24$ yo ( $p>0.2$ both sexes). Diabetes mortality did not significantly change in the EU15 + across the study period in any age group. In contrast, amongst 1-14 year olds the UK had a significantly higher rate of decline than the EU15+ (negative slope coefficient, $\mathrm{p}=0.03$ ). UK mortality rose amongst $15-24$ year olds compared with the EU15+ (positive slope coefficients, p < 0.0001 both sexes) In 1990, the UK had higher diabetes mortality than the US in 1-14 year olds ( $\mathrm{p}<0.0001$ ) but lower amongst $15-24$ year olds ( $\mathrm{p}<0.0001$ both sexes). The USA had little change in diabetes mortality amongst 1-14 year olds but a significant rise in diabetes mortality amongst $15-24$ year olds ( $p<0.0001$ both sexes). The UK had a greater rate of mortality decline that the USA amongst 1-14 year olds (negative slope

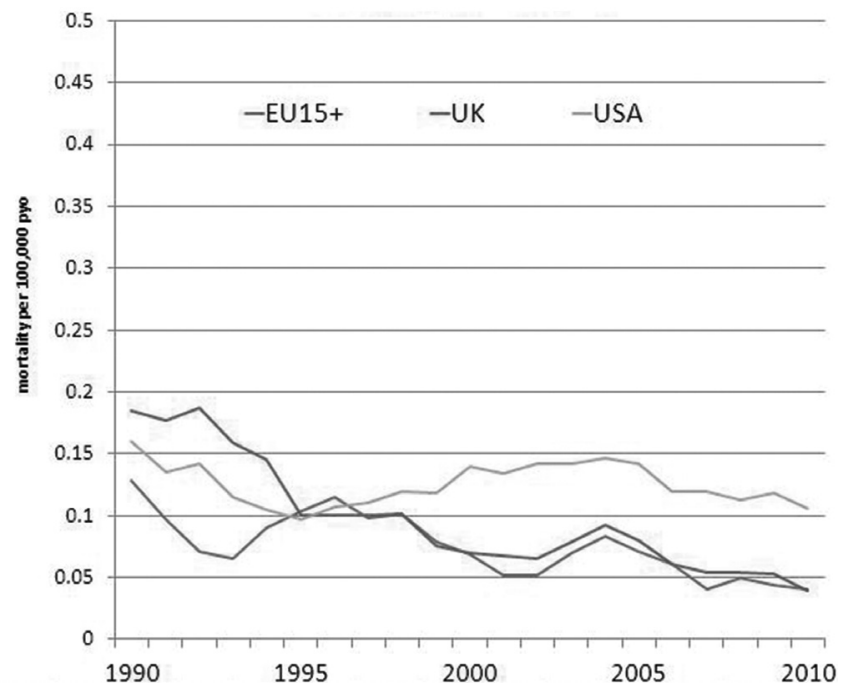

Abstract G476 Figure 1 Total diabetes mortality amongst 1-14 year olds (3 year moving averages) 